\title{
Interface Modes in Semiconductor Terahertz Layer Structures
}

\author{
T. Gric \\ Vilnius Gediminas Technical University, Vilnius, Lithuania \\ tatjana.gric@dal.ca; tatjana.gric@gmail.com
}

\begin{abstract}
We address and explain the occurrence of interface modes in GaAs/AlAs semiconductor layer structures. Interface modes are tied to the existence of a surface, i. e. interface between two media of certain dielectric properties. We have assigned the transverse-magnetic interface modes by solutions of the surface polariton dispersion relations for semiconductor layer structures. Derivations employ full phenomenological electromagnetic description and are based on fundamental defining equations of the electromagnetic field and wave propagation theory.
\end{abstract}

OCIS Codes: (240.6680) Surface plasmons; (240.5420) Polaritons.

PACS: $73.20 . M f, 71.36+c$.

\section{INTRODUCTION}

Presently, microelectronics and photonics are the fundamental technologies which are the pivot of the modern science and engineering.

Photonic devices can operate at the high frequencies. Thus, there are the theoretical possibilities to increase the speed of information processing in optical computers by a factor of $\sim 10^{5}$ or more, compared to conventional computers based on microelectronics. Photonic devices, such as optical fibers, couplers and switches have already been successfully integrated into telecommunication and computer networks [1].

The advantages of photonic devices over microelectronic devices come due to the fact that the carriers of information are photons rather than electrons $[1,2]$. Unfortunately, photonic devices are much larger than the microelectronic ones. The reason is the diffraction limit, which theoretically limits the minimum sizes of photonic devices (for example, the core diameter of an optical fiber) to around half wavelength $[1,2,3,4]$.

Nevertheless, microelectronics has already been miniaturized, but still the problem of manufacturing at the nanometer scale exists. Also, the fundamental problems related to heat or interference with external electromagnetic (EM) fields should be mentioned [5]. Consequently, the essential problem of the size mismatch between photonic and microelectronic devices remains unsolved [6, 7].

In order to solve the above mentioned problem we have to deal with the EM surface waves on metallic interfaces, called surface plasmons (SP) or just plasmons. Surface plasmon research, also known as plamonics, is a new branch of nanophotonics or nano-optics $[2,8,9]$.

It should be pointed out that SP's can be employed not only on metals but also on doped semiconductors [10]. The layers with different alloy compositions are used to construct the semiconductor device structures with individual dopant incorporation. The modern non-equilibrium growth techniques are employed to grow the novel metastable materials, unavailable from natural 


\section{T. Gric}

sources. It is mandatory to determine their physical properties seeking to obtain the appropriate design of hetero structure devices. The adequate experimental techniques are required to control and characterize the individual layer properties during and after deposition.

The frequency dependent complex-valued dielectric function $\varepsilon$ describes the link between the internal linear interactions (polaritons) [11] of light with matter and the externally observable changes of a probing electromagnetic radiation field sintensity, polarization stated. The additional interface-bound polaritons, which descend from the bulk-supported polaritons of the adjacent media are imposed by the hetero interfaces. The polaritons involve lattice excitations with polar momentum and free-chargecarrier plasma modes. The resonant excitation of plasmon- supported bulk and interface modes (surface polaritons, surface guided waves (SGW)) strongly effect the optical response of layered structures composed of polar semiconductor materials. One can obtain the detailed information on plasmon mode parameters from model analysis of the frequency-dependent sample response.

The design of terahertz structures is a hot research topic during the past years. Due to the possibility of sub-wavelength confinement and field enhancement [12], these structures can be used in a large variety of applications ranging from thin film spectroscopy [13] to high resolution imaging below the diffraction limit [14]. One can come up with the analysis of such waveguides using finite differencetime-domain methods, which yield the field distribution and mode profile inside the waveguide. However, in this case the problems are encountered at $\mathrm{THz}$ frequencies when metals or highly doped semiconductors are involved and the permittivity of neighboring layers differs by several orders of magnitude. Thus, a very fine discretization is required that leads to a correspondingly higher demand on computational resources.

In this paper, we discuss modes in semiconductor layer structures. We assign modes by solutions of the surface polariton dispersion relations for polar semiconductor layer structures. The aim of the present work is to demonstrate the physical origin of surface modes in semiconductor layer structures. Our contribution is to present a simple analytical algorithm which makes is possible to include in the analysis other than metallic media. It should be pointed out, that any prior knowledge about the dispersion relation or the mode structure is not required. A theoretical treatment is based on the fundamental defining equations of the electromagnetic field and wave propagation theory and results in the new solution for an optical plasmon at a semiconductor/semiconductor interface.

\section{Polaritons in Semiconductor Layer Structures}

In case of doped GaAs, the electric function $\varepsilon(\omega)$ is contributed by the free charge-carriers. The classical Drude equation is valid for description of $\varepsilon(\omega)$. For single free charge-carriers,

$$
\varepsilon(\omega)=\varepsilon_{\infty}-\frac{\omega_{p}^{2}}{\omega\left(\omega+i \gamma_{p}\right)}
$$

The unscreened plasma frequency can be expressed as $\omega_{p}=e \sqrt{N /\left(\varepsilon_{0} \varepsilon_{\infty} m\right)}$, where $N$ is the free-chargecarrier concentration, and $m$ is the effective mass ( $\varepsilon_{0}$ is the vacuum permittivity and $e$ is the electrical unity charge), $\varepsilon_{\infty}$ - is the dielectric constant of the material.

The phenomena involving resonant propagation of EM waves and energy transport parallel to the surface is addressed by the term surface mode. SGW refer to the waves with transverse-electric (TE; electric field vector parallel to interface) radiation fields. The total internal reflection and guided slab 
modes are some of the examples. Surface polaritons are interface-bound EM waves of transversemagnetic (TM; magnetic field vector parallel to interface) character.

It is impossible to excite the surface polaritons at a single interface within a standard reflection or transmission experiment. It happens due to the allowed dispersion range which is to the right of the "light line" $\omega=c k$. Therefore, the high-index-of-refraction prisms are necessary to increase the wave vector of the incident light wave. Though, the boundary conditions for certain wavelengths, where resonant excitation of SP's can be observed without use of a high-index prism can be produced by the dielectric properties of polar semiconductors at long wavelengths, bound within a thin-film structure.

In this work we demonstrate the occurrence of SP modes in polar semiconductor heterostructures. A brief derivation of the single- interface polariton dispersion relations is presented in this section. The subsequent discussion holds for polar heterostructures as well (e.g., GaAs-AlAs). In order to obtain the better visibility of the SP effects within the calculated spectra we have chosen the plasma broadening values that correspond to very high carrier mobility parameters.

The starting equations, i. e. Maxwell's equations, the charge continuity equation, and the electron motion equation are notoriously known in the literature [15]. In order to derive the dispersion relation, we need to satisfy the boundary conditions for fields in both media. At the end we get the dispersion relation in the form:

$\omega^{2}\left(\varepsilon_{1}-\varepsilon_{2}\right)+\beta^{2} c^{2}\left(\frac{\varepsilon_{2}}{\varepsilon_{1}}-\frac{\varepsilon_{1}}{\varepsilon_{2}}\right)=0$

To begin with, the solution of Eq. 2 is discussed for the $\operatorname{GaAs}\left(\varepsilon_{1}\right)-\operatorname{AlAs}\left(\varepsilon_{2}\right)$ interface. The dielectric functions are as follows:

$$
\begin{aligned}
& \varepsilon_{1}(\omega)=\varepsilon_{\infty 1}-\frac{\omega_{p 1}^{2}}{\omega\left(\omega+i \gamma_{p 1}\right)}, \\
& \varepsilon_{2}(\omega)=\varepsilon_{\infty 2}-\frac{\omega_{p 2}^{2}}{\omega\left(\omega+i \gamma_{p 2}\right)},
\end{aligned}
$$

Where $\varepsilon_{\infty 1}, \varepsilon_{\infty 2}$ denote GaAs and AlAs dielectric constants. $\varepsilon_{\infty 1}=12.9, \varepsilon_{\infty 2}=10.1$. It is worthwhile to notice, that the permittivities of both semiconductors are assumed lossless; hence $\varepsilon_{1}$ and $\varepsilon_{2}$ are real throughout this work.

Eq. 2 has four physical roots,

$$
\omega_{1,2}= \pm \sqrt{2} \cdot\left(\begin{array}{l}
1 / \sqrt{\left(\begin{array}{l}
\left(\begin{array}{l}
\beta^{4} c^{4} \varepsilon_{\infty 1}^{2}+2 \beta^{4} c^{4} \varepsilon_{\infty 1} \varepsilon_{\infty 2}+\beta^{4} c^{4} \varepsilon_{\infty 2}^{2}+2 \beta^{2} c^{2} \varepsilon_{\infty 1}^{2} \omega_{p 2}^{2}- \\
-2 \beta^{2} c^{2} \varepsilon_{\infty 1} \varepsilon_{\infty 2} \omega_{p 1}^{2}-2 \beta^{2} c^{2} \varepsilon_{\infty 1} \varepsilon_{\infty 2} \omega_{p 2}^{2}+2 \beta^{2} c^{2} \varepsilon_{\infty 2}^{2} \omega_{p 1}^{2}+ \\
+\varepsilon_{\infty 1}^{2} \omega_{p 2}^{4}-2 \varepsilon_{\infty 1} \varepsilon_{\infty 2} \omega_{p 1}^{2} \omega_{p 2}^{2}+\varepsilon_{\infty 2}^{2} \omega_{p 1}^{4}
\end{array}\right)+ \\
+\varepsilon_{\infty 1} \omega_{p 2}^{2}+\varepsilon_{\infty 2} \omega_{p 1}^{2}+\beta^{2} c^{2} \varepsilon_{\infty 1}+\beta^{2} c^{2} \varepsilon_{\infty 2}
\end{array}\right)} \\
\times \sqrt{\left(\beta^{2} c^{2} \omega_{p 1}^{2}+\beta^{2} c^{2} \omega_{p 2}^{2}+\omega_{p 1}^{2} \omega_{p 2}^{2}\right)}
\end{array}\right),
$$




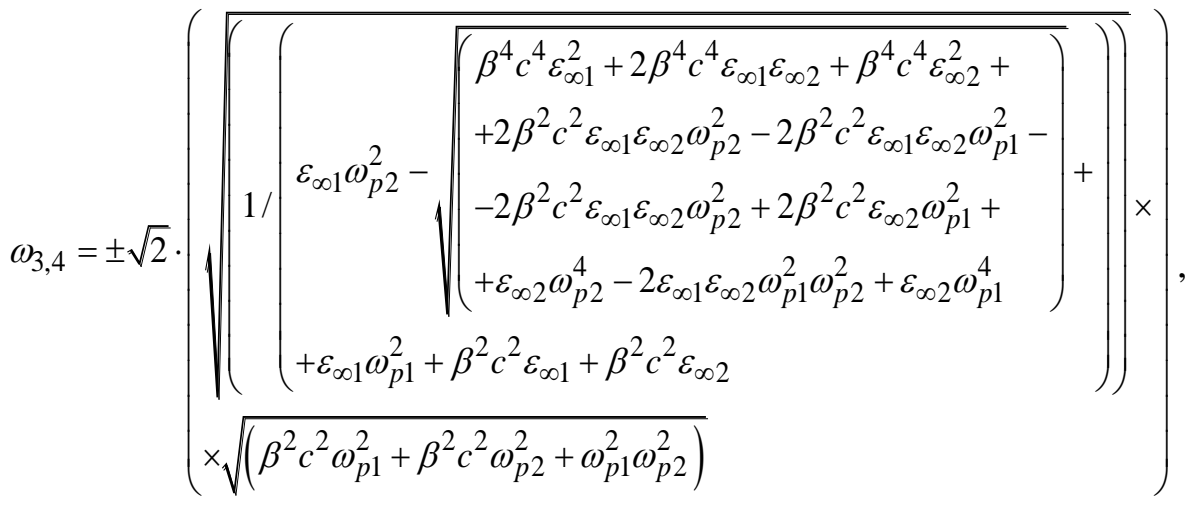

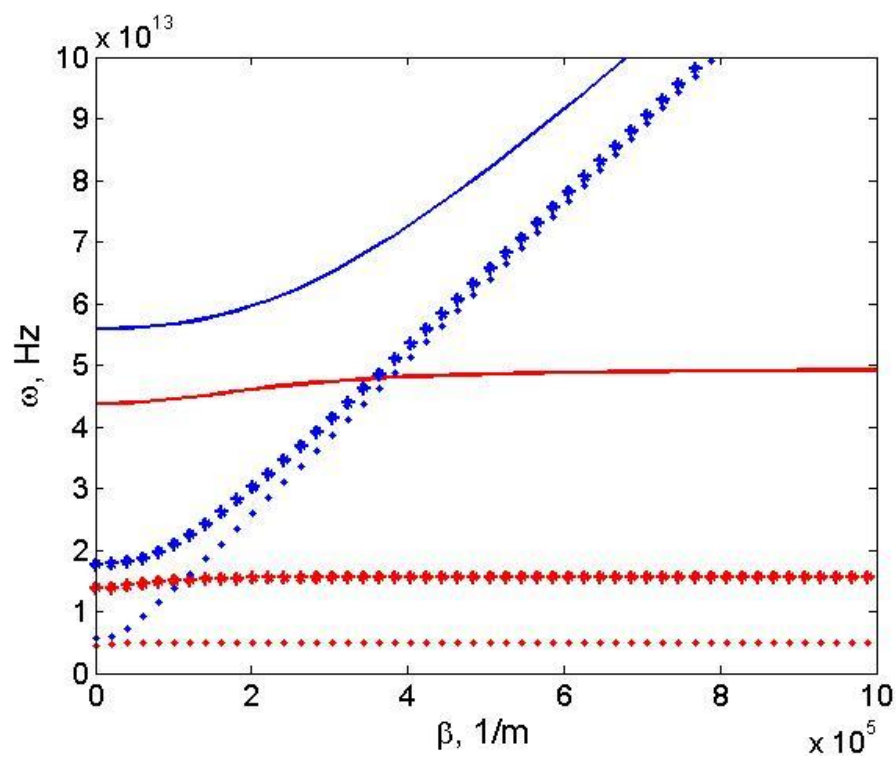

Fig1. SP dispersions for the GaAs/AlAs interface.

It is worthwhile to notice, that the mode $\omega_{3}$ possesses strong dispersion, although $\omega_{1}$ is bound within a very small frequency region. Figure 1 depicts the SP dispersions. It should be pointed out, that $\omega_{3}$ and $\omega_{1}$ can be described by Eq. (5) and Eq. (4), respectively. The modes depicted by the solid lines correspond to the concentration $N=10^{26} \mathrm{~m}^{-3}$, by the stars - to the concentration $N=10^{25} \mathrm{~m}^{-3}$, by the dots - to the concentration $N=10^{24} \mathrm{~m}^{-3}$. Also, it is worthwhile to mention, that modes $\omega_{3}$ are presented by blue color, and the modes $\omega_{1}-$ by red color. The effective mass of GaAs is $0.067 m_{e}$ and the effective mass of AlAs is $0.15 m_{e}[16]$.

A boundary of two different semiconductors is probably one of the most basic structures to describe SP propagation. Nevertheless, some important results can be derived with this simple configuration. Within this work, it is assumed that the interface, which separates two semiconductors, is placed on the $x-y$ plane of a Cartesian coordinate system. It is also assumed that the GaAs occupies the region $z<0$ and the SP's propagate along the $x$-axis. It can be demonstrated that only TM-plasmons can propagate along the surface. An explanation could be derived from the boundary condition

$\left(D_{s 1}-D_{s 2}\right) \cdot n=\delta$,

Where $D_{s 1}$ and $D_{s 2}$ are the dielectric displacement in the GaAs and AlAs, respectively and $n$ is the normal vector at the interface. As a consequence the longitudinal surface charge density oscillations in the direction of propagation ( $x$-axis) can be induced only by the components of $E$ in the plane of the incident wave. The only magnetic field component left is $H_{y}$, therefore only TM-plasmon can 
propagate along interface of two semiconductors. The EM field associated with TM-plasmons, also decay exponentially away from the interface into two both media, see also Fig. 3. Hence, the solution for $H_{y}$ can be presented as:

$$
H_{y}=A e^{j(\omega t-\beta x)} \cdot \exp \left(\alpha_{1} z\right) z<0, \mathrm{GaAs}
$$

And

$$
H_{y}=B e^{j(\omega t-\beta x)} \cdot \exp \left(-\alpha_{2} z\right) z>0, \mathrm{AlAs},
$$

Where $A$ and $B$ are amplitudes to be determined by the boundary conditions, $\alpha_{1}$ and $\alpha_{2}$ are the reciprocal (positive and real) penetration depths into the GaAs and AlAs, respectively. The amplitude of SP's decreases with increasing distance and eventually dissipates. The penetration depth is defined as the distance from the interface GaAs-AlAs at which the amplitude of $H_{y}$ is reduced by $e^{-1}$ :

$$
\alpha_{1 / 2}=\sqrt{\beta^{2}-k_{0}^{2} \varepsilon_{1 / 2}} \text {. }
$$

From (9) one can make a conclusion that the penetration depth of the plasmon into the GaAs is smaller in to the AlAs due to the fact, that $\varepsilon_{\infty 1}>\varepsilon_{\infty 2}$. The graphical visualization is presented in Fig.2.

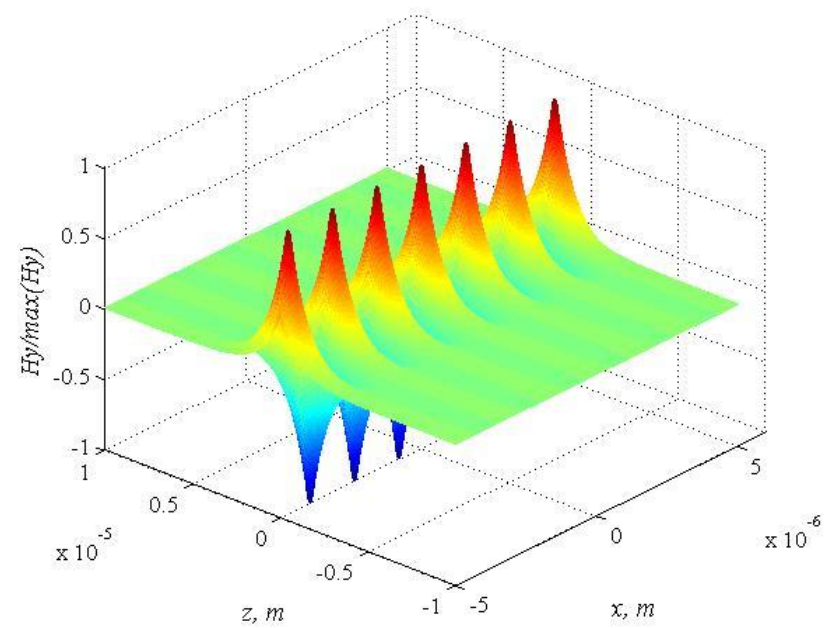

Fig2. 3D-model of a surface plasmon, $\omega_{1}$ mode propagating along an interface of two semiconductors in the $x$ direction. Schematically a snap shot of the $H_{y}$ distribution (TM-mode) is show.

\section{INAS-AIR BOUNDARY}

In order to validate our procedure, the dispersion relation of surface waves coupled to surface carriers on a vacuum to InAs boundary using (Eq. 2) has been calculated. Mead and Spitzer [17] have come to a conclusion, that InAs shows a native inversion layer on the surface due to pinning of the Fermi level inside the conduction band. The thickness of this inversion layer is strongly reduced when the InAs is $p$-type doped and can reach values as low as several $100^{\circ} \mathrm{A}$ [18]. Moreover, the 2-D nature of the electrons confined to the surface layer has been confirmed by magneto resistance measurements [19]. Due to this native inversion layer, bulk InAs can be used as THz emitter and it has been shown that the $\mathrm{THz}$ emission from an irradiated InAs surface in a magnetic field can be attributed to 2-D magneto-plasma oscillations [20]. 


\section{T. Gric}

We use the same parameters as in [22] to be able to compare with literature. Consequently, the various effects such as impurity scattering and free carrier absorption have been neglected. However, the generality of the presented method is not influenced by the choice of the values of the dielectric functions and one can include these effects in the model without facing any difficulties. We use $\varepsilon_{1}(\omega)$ $=1$ for vacuum and the dielectric function of $p$-type InAs described by the Drude model (Eq. 1). It is assumed, that $\varepsilon_{\infty}=11.8$, and $\omega_{p}=2.95 \times 10^{13} \mathrm{~s}^{-1}$ is the free-carrier plasma frequency corresponding to a hole concentration of $N=7 \times 10^{16} \mathrm{~cm}^{-3}$.

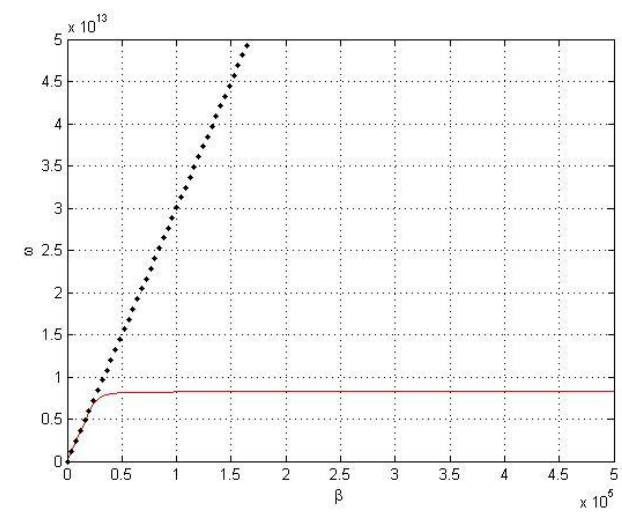

Fig3. Dispersion relation for SWC on a clean surface of p-type InAs. The dots represent the vacuum lightline and the dashed line the bulk polariton dispersion. The solid line represents the calculated dispersion relations of surface waves.

Fig. 3 shows the dispersion of the surface waves. The case corresponds to classic surface waves as are well known for conductive interfaces [21]. In addition, the lightline in vacuum is plotted in Fig. 3 as dotted line. Our calculated data corresponds to the data of [22] where also a complete discussion of the surface wave characteristics in this case can be found.

This example illustrates how the dispersion in semiconductor/air structure can easily be calculated by our method.

\section{SUMMARY}

In summary, a simple and robust method for the calculation of the dispersion relation of modes in planar structures has been presented. By analytically deriving equations for the modes, it is possible to come up with the calculations of the structures in the $\mathrm{THz}$ frequency range with very good convergence behavior. It should be pointed out, that no a priori knowledge about the dispersion is necessary. Moreover, due to the reason, that the derivation of the formulas has not been based on special forms of dielectric functions, the method is assumed to be valid for a broad range of possible structures. Furthermore, following the proposed methodology the radiative and bound modes can be calculated by means of the derived equations (Eqs. 4, 5). Thus, we are avoiding the problem of appearance of a frequency gap region with purely imaginary propagation constant $\beta$ prohibiting propagation. In other words, the presented mathematical model serves a convenient system to examine both radiative and bound modes. This has been demonstrated on the example which is of current interest in THz device technology.

\section{REFERENCES}

[1] R. G. Hunsperger, Integrated Optics: Theory and Technology, Springer, $6^{\text {th }}$ ed. 2009.

[2] P. N. Prasad, Nanophotonics, Wiley 2004.

[3] D. F. P. Pile, D. K. Gramotnev, Appl. Phys. Lett. 86(16), 161101 (2005).

International Journal of Research Studies in Electrical and Electronics Engineering (IJRSEEE) Page | 26 
[4] D. F. P. Pile, D. K. Gramotnev, Opt. Lett. 30(10), 1186-1188 (2005).

[5] S. Kawata, M. Ohtsu, M. Irie, Nano-Optics, Springer, 2002.

[6] M.L. Brongersma, R. Zia, J.A. Schuller, Appl. Phys. A 89(2), 221-223 (2007).

[7] E. Ozbay, Science 311(5758), 189-193 (2006).

[8] H. A. Atwater, Sci. Am. 17, 56 - 63 (2007).

[9] W. L. Barnes, A. Dereux, T. W. Ebbesen, Nature 424, 824-830 (2003).

[10] M. S. Kushwaha, Surf. Sci. Rep. 41(1-8), 1-416 (2001).

[11] J. J. Hopfield, Phys. Rev. 112, 1555 (1958).

[12] J. A. Dionne, L. A. Sweatlock, H. A. Atwater, A. Polman, Phys. Rev. B 73(3), 035407 (2006).

[13] T. H. Isaac, W. L. Barnes, E. Hendry, Appl. Phys. Lett. 93(24), 241115 (2008).

[14] M. M. Awad and R. A. Cheville, Appl. Phys. Lett. 86(22), 221107 (2005).

[15] M. Cada, J. Pistora, "Optical plamonics in semiconductors," ISMOT conference, June 20-23, 2011.

[16] S. Adachi, Properties of Aluminium Gallium Arsenide, INSPEC; The Institution of Electrical Engineers, London 1993.

[17] C. A. Mead, W. G. Spitzer, Phys. Rev. Lett. 10(11), 471-472 (1963).

[18] F. Stern and W. E. Howard, Phys. Rev. 163(3), 816-835 (1967).

[19] S. Kawaji and Y. Kawaguchi, J. Phys. Soc. Jpn. Suppl. 21, 336-340 (1966).

[20] J. N. Heyman, P. Neocleous, D. Hebert, P. A. Crowell, T. M“uller, K. Unterrainer, Phys. Rev. B 64(8), 085202 (2001).

[21] S. A. Maier, in Plasmonics: Fundamentals and Applications, Springer: New York, 2007, ch. 2, pp. 21-37.

[22] M. Nakayama, J. Phys. Soc. Jpn. 36(2), 393 (1974. 